\title{
Therapeutic Effect of BCG Polysaccharide Nucleic Acid Combined with Traditional Chinese Medicine in the Treatment of Multiple Foot Wounds with Hand - Foot
}

\author{
Qingfeng Wan ${ }^{1}$, Chunhua Yü ${ }^{2}$, Wenbin Zhang ${ }^{3}$ \\ ${ }^{1}$ Jiangxi Medical Collage, Shangrao, Jiangxi, China, 334000 \\ ${ }^{2}$ Jiangxi Medical Collage, Shangrao, Jiangxi, China, 334000 \\ ${ }^{3}$ Guangfeng People’s Hospital,Shangrao, Jiangxi, China, 334600
}

Keywords: Hand-Foot Frequent Warts, Chinese Medicine Topical, BCG Polysaccharide Nucleic Acid

\begin{abstract}
Warts" by our clinical medicine is classified as a human papillomavirus infection caused by mucosal, skin new creatures, which usually include warts, genital warts and flat warts, this study uses the combination of traditional Chinese and Western medicine in the treatment on the hand and foot multiple sexual warts, reported as follows.
\end{abstract}

\section{Introduction}

Ordinary warts appear mainly in the hands of patients, commonly known as "child", "thorns", Chinese medicine called "thousands of sore", the disease mainly by the human papillomavirus (the virus type is $1,2,4,7,27,28,48,63$ ) infection, the patient may also have a certain degree of immune problems. For some parts of the palm plantar, a week and other parts of the common warts patients, conventional treatment methods are mainly liquid nitrogen freezing and carbon dioxide laser, etc., and physical therapy methods are repeated, severe pain and other defects.

The aim of this study was to investigate the efficacy of BCG polysaccharide nucleic acid combined with traditional Chinese medicine in the treatment of multiple foot wounds. Methods: 67 cases of warts were divided into control group and observation group. Among them, 32 cases were divided into control group and 35 cases were divided into observation group. In the control group, the patients were treated with traditional Chinese medicine. The observation group was treated with BCG polysaccharide nucleic acid combined with traditional Chinese medicine. The treatment results were compared between the two groups. Results: The control group and the experimental group and the effective rate of treatment were $50 \%$ and $74.3 \%, \mathrm{P}<0.05$ was statistically significant. Conclusion: The application of BCG polysaccharide nucleic acid combined with traditional Chinese medicine in the treatment of hand, foot and multiple warts can effectively improve the efficiency of treatment, and there is no adverse reaction in any form. The clinical value should be popularized.

\section{General Information}

The 74 samples selected from this study were rigorously diagnosed according to the criteria of disease diagnosis and typical symptoms. Focus on the study of patients with hand and foot lesions, in the statistical treatment and clinical observation of the link is not included in other parts of the skin lesions. 74 cases of study samples were multiple lesions, the number of lesions in the 3 to 15 , the lesion shape for the polygonal or round, the tip to the pea size, touch hard, rough surface, the surface was brown, dirty yellow and grayish yellow. Skin and palmoplantar parts are basically flat were nipple-like proliferation and higher than the leather surface. All of the study samples did not undergo any drug treatment for the disease within 1 month prior to treatment. I included patients with infectious diseases, severe systemic diseases, and patients with immune diseases. There was no form of allergic dermatitis, eczema, etc. 7 patients in the treatment process to give up treatment, not 
its efficacy evaluation, only for safety evaluation. The remaining 67 patients were included in the statistical analysis, which was divided into two groups: control group and observation group. Among them, 32 cases were divided into control group and 35 cases were divided into observation group. There were 18 cases of female patients and 17 cases of male patients. The age was 63 years old and the youngest was 15 years old. The average age was $29.67 \pm 14.78$ years. The course was 16 years, the shortest course was 1 year, and the average duration was 7.84 .52 years. There were 16 cases of female patients and 16 cases of male patients. The age was 62 years old and the youngest was 16 years old. The average age was $30.41 \pm 13.65$ years old. The course was 17 years, the shortest course was 2 years, and the average duration was $7.75 \pm 4.32$ years. There was no significant difference in the course of disease, age and sex between the two groups ( $\mathrm{P}>0.05)$.

\section{Method}

Nurses in the treatment of patients before the need to inform patients in advance of traditional Chinese medicine treatment may occur in a variety of adverse reactions, and encourage patients to overcome difficulties, adhere to long-term treatment. Inform the patient need to pay attention to the relevant matters, to avoid the problem of infection.

Observation Group. In this study, 35 patients were treated with BCG polysaccharide nucleic acid injection combined with traditional Chinese medicine treatment, nursing staff to provide BCG polysaccharide nucleic acid intramuscular injection, once every 2 days, each injection $2 \mathrm{ml}$, joint indicators $30 \mathrm{~g}$ of horseradish, $30 \mathrm{~g}$ of horseradish, $30 \mathrm{~g}$ of honeysuckle, $30 \mathrm{~g}$ of hibiscus, $30 \mathrm{~g}$ of Speranskia, 30g Hedyotis diffusa, boiled time For 30 minutes to decoction outside the soup, the daily topical 1 , the patient after daily medication, by the patient can also be used before the sterilization of the blade wart body stool and wart surface cut off the surface. Nursing staff need to guide the patient the correct blade operation method to avoid misunderstanding, bleeding and other aspects of the problem, once the patient scraping the skin, you need to immediately use hydrogen peroxide and iodine disinfection, do a good job dressing. At the same time, the blade used by the patient also needs a disposable blade for medical use, prohibiting the patient from using the blade repeatedly.

Control Group. The patients in the control group were treated with the traditional Chinese medicine topical therapy, the precautions, the treatment time and the treatment method. It until the patient completely dissipated after the termination of treatment.

Statistical Analysis. All the data obtained by this method are processed by SPSS 17.0 software. When $\mathrm{P}>0.05$, the difference was not statistically significant; when $\mathrm{P}<0.05$, the difference was statistically significant. With $\chi^{2}$ test to compare the function and symptoms; $\pm \mathrm{s}$ used to represent the measurement data, using t test.

\section{Observation of Efficacy}

Evaluation Criteria for Efficacy. Nursing staff received out-of-hospital follow-up to receive the patient in the treatment of the first month, February and March of the symptoms of recovery, and pre-treatment disease performance were compared. Efficacy evaluation criteria were divided into invalid, effective, markedly and cured 4 grades. Effective: to obtain $30 \%$ to $60 \%$ of the skin lesions regression rate; markedly: get more than $60 \%$ of the skin rate of withdrawal; recovery; skin lesions completely subsided. The total effective rate is the sum of the number of people and the number of people recovered, and the total effective rate is the ratio of the total effective number to the total number of samples. 


\section{Results.}

Table 1 The observation group

\begin{tabular}{|l|c|c|c|c|c|c|}
\hline Treatment & Invalid & Effective & Effective & Get Well & Total & $\begin{array}{c}\text { Efficient } \\
(\%)\end{array}$ \\
\hline The First Month & 4 & 18 & 10 & 3 & 35 & 37.14 \\
\hline The Second Month & 3 & 13 & 15 & 7 & 35 & 54.29 \\
\hline The Third Month & 1 & 8 & 17 & 9 & 35 & 74.30 \\
\hline
\end{tabular}

Table 2 The control group

\begin{tabular}{|l|c|c|c|c|c|c|}
\hline Treatment & Invalid & Effective & Effective & Get Well & Total & $\begin{array}{c}\text { Efficient } \\
(\%)\end{array}$ \\
\hline The First Month & 8 & 16 & 6 & 2 & 35 & 25.00 \\
\hline The Second Month & 6 & 13 & 8 & 5 & 35 & 40.63 \\
\hline The Third Month & 4 & 12 & 9 & 7 & 35 & 50.00 \\
\hline
\end{tabular}

The difference between the two groups of patients in the control group and the observation group was significant $(\mathrm{P}<0.05)$.

Comparison of Adverse Reactions. In this study, 74 cases of anti-selected samples, half-way out of the experiment in 7 cases, including 1 case of female patients, 2 cases of children, the reason is due to BCG polysaccharide nucleic acid injection caused by swelling and pain, the rest 4 cases of patients with withdrawal from the middle of one case of women and all adult patients, the reasons are unable to adhere to long-term self-medication for external use. The presence of localized itching in the treatment of 2 patients in the 67 samples of this study showed that the caregiver appropriately reduced the soaking time and continued to adhere to the treatment.

\section{Discussion and Conclusions}

According to the current epidemiological data has been able to fully confirm the widespread existence of human papillomavirus infection in China's population, easily lead to skin and mucous membrane infection, and then lead to common warts, common warts are the main symptoms of skin and mucous membrane infection, adolescents and children Is the main incidence of the population. Clinical medicine in the treatment of common warts are many ways, usually including surgical resection, freezing, microwave and laser and other physical therapy methods, but the physical treatment on the one hand only palliative, on the other hand in the range of lesions and depth cannot be accurate The patient after the completion of treatment may be in a long period of time to leave scars in the patient after the completion of treatment, the wart surface will be necrotic and shedding and wart body tissue there is still some residual, causing disease recurrence. Laser therapy is a typical creative treatment, but cannot be applied to the plantar, joints and limbs and other parts of the relevant postoperative care are also very complex. For the treatment of multiple warts, especially the joint parts, a week parts of the common warts patients, the choice of treatment options are very limited. In addition, for most children, patients need to consider in the specific treatment to the pain caused by children. The relevant treatment means also need to ensure that patients without any vegetation residues and reproduction, to avoid adverse effects on the skin.

The results of multi-party investigation and experimental study found that traditional Chinese medicine treatment methods have an irreplaceable advantage for the common warts, the focus of this experiment to join the traditional Chinese medicine treatment methods for treatment group of patients treated. Chinese herbal medicine warts contained in the honeysuckle, Hedyotis diffusa and horseshoe grass ingredients can play antiviral and detoxification and detoxification and role; Prunella vulgaris, Speranskia and Cyperus rotundus can play the role of liver qi channel ; Mulberry leaves can play a role in improving blood circulation, warm and cold; safflower can play the role of blood qi, blood circulation, for refractory, multiple common warts have a very good therapeutic effect, the use of traditional Chinese medicine treatment Method to improve the role of the patients at the same time also eliminates the pain in the physical treatment process, effectively improve the 
treatment of children with patient compliance. However, the treatment of external medicine, but also the existence of long treatment cycle, patients need to boil Chinese medicine and other aspects of the lack of this experiment, 4 patients due to the treatment operation is very complex way out of the experiment.

The experiment also joined the intramuscular injection of BCG polysaccharide nucleic acid treatment, Chinese medicine topical treatment once a day, Western medicine injection treatment every 2 days. BCG polysaccharide nucleic acid is a good quality bidirectional immunomodulator. In the treatment of multiple warts, the mechanism of action of the drug is to stimulate the body's endothelial system within the body, so that mononuclear macrophages in a relatively short period of time to activate, play a humoral immune and cellular immune function regulation The role of natural killer cells through the way to improve the patient's own anti-virus ability. Multidisciplinary investigation and experimental study found that BCG polysaccharide nucleic acid through the central immune organs, peripheral immune organs on CD8 + cells and CD4 + cell differentiation and maturation of direct regulation, so that the two balance, and thus improve the immune function of patients. The results of this experimental study, the control group and the experimental group and treatment efficiency were $50 \%$ and $74.3 \%, \mathrm{P}<0.05$ was statistically significant. It is effective to provide BCG polysaccharide nucleic acid combined with traditional Chinese medicine for the treatment of hand, foot and multiple warts. It can effectively improve the efficiency of treatment. There is no adverse reaction in any form. The clinical value is worth popularizing.

In the specific treatment of the part, the patient in the routine treatment of traditional Chinese medicine, the patient can be prepared in advance by the disposable medical special blade wart body stool and wart surface cuticle clean play the role of disease rehabilitation, consolidate the treatment effect.

Because of the small number of samples selected in this experimental study, the selected results may have some limitations and randomness. At the same time, the recurrence of common warts and long-term problems of recurrence of patients also need medical staff to conduct in-depth study, if conditions permit, after the investigation and experiment also need to further expand the number of research samples, but also need to strengthen the recurrence of patients and treatment The correlation between the methods of analysis, to improve the experimental results of persuasive and scientific.

\section{References}

[1] Zhang Xunjing. Chinese herbal medicine combined with Mycobacterium phlei injection for the treatment of multiple warts [J]. Modern Journal of Integrated Traditional and Western Medicine, 2013, 05 (08): 1623-1624.

[2] Yu Ye, Sun Yan, Tang Ye. BCG polysaccharide nucleic acid combined with traditional Chinese medicine treatment of hand, foot and multiple warts worsening effect [J]. Sichuan Traditional Chinese Medicine, 2013, 10 (26): 75-77. 